\title{
Computer Vision based Defect Detection and Identification in Handloom Silk Fabrics
}

\author{
R. S. Sabeenian \\ Professor - ECE \& Centre \\ Head - SONA SIPRO, \\ Advanced Research Centre, \\ Sona College of Technology, \\ Salem, Tamil Nadu, INDIA
}

\author{
M. E. Paramasivam, \\ Assistant Professor - ECE \& \\ Team Member Sona SIPRO \\ Advanced Research Centre, \\ Sona College of Technology, \\ Salem, Tamil Nadu, INDIA.
}

\author{
P. M. Dinesh \\ Assistant Professor - ECE \& \\ Team Member Sona SIPRO \\ Advanced Research Centre, \\ Sona College of Technology, \\ Salem, Tamil Nadu, INDIA.
}

\begin{abstract}
Fabric defect detection and classification plays an important role in inspection of fabric products. Many fabric defects are very small and undistinguishable, which can be detected only by monitoring the variation in the intensity. Currently, in almost all the fabric industries the process of defect detection is done manually using skilled labor. An automated defect detection and identification system would naturally enhance the product quality and result in improved productivity to meet both customer demands and also reduce the costs associated with off-quality.
\end{abstract}

The main objective of this proposed work is to check whether the fabric material is defective or not, if defective, then identify the location and type of the defect. This paper deals with the defect detection process using Multi Resolution Combined Statistical and Spatial Frequency (MRCSF), Markov Random Field Matrix method (MRFM), Gray Level Weighted Matrix (GLWM) and Gray Level Co-occurrence Matrix (GLCM).

\section{General Terms}

Multi Resolution Combined Statistical and Spatial Frequency (MRCSF), Markov Random Field Matrix method (MRFM), Gray Level Weighted Matrix (GLWM) and Gray Level Cooccurrence Matrix (GLCM)

\section{Keywords}

Defect Detection in Silk Fabrics, Pattern Recognition,

\section{INTRODUCTION}

One of the most important industries, which is dominating today's world is the textile industry, with almost numerous fascinating products being produced every minute to satisfy the needs of the customers. Fabrics of various kinds and many designs are very much attracted by people irrespective of caste, creed and nation. Fabric industry can be considered as an evergreen industry, where in there are no rises and falls which can be affected either by the global or national sensex.

Fabrics, in olden days were hand-woven by making use of hand-twinned cotton. Even today, some delicate threads such as silks from silk worms are preferred to be hand-woven rather than machine-woven. Now-days, power-looms have dominated a lot on handloom, as a result of which the Government of India along with the respective State Governments gives subsidiaries to the weavers, co-operative societies handling handloom fabrics and in certain special cases, even the Sericulture Department.
Handlooms constitute a rich cultural heritage of India. The handloom weaving, as an economic activity, provides livelihood to many people. The element of art and craft present in Indian handlooms makes it a potential sector for the upper segments of market in domestic as well as global. The sector accounts for $13 \%$ of the total cloth produced in the country [1]. The strength of Handloom lies in introducing innovative design, which cannot be replicated by the powerlooms. Innovative weavers with their skillful blending of myths, faiths, symbols and imagery provide their fabric an appealing dynamism. In spite of the Government intervention through financial assistance and implementation of various development and welfare schemes, the number of handlooms is continuously reducing in the country. The reasons are manifold. New generations are not readily joining the weaving activity, low wages, continuous increase in yarn prices, obsolete technologies, unorganized production system, low productivity, inadequate working capital, conventional product range, weak marketing link, overall stagnation of production and sales and, above all, competition from powerloom are the factors forcing the handloom sector difficult to survive.

Handloom industry in Tamil Nadu plays an important role and provides employment for more than 4.29 lakh weaver households and about 11.64 lakh weavers [1]. According to the Director of Handlooms \& Textiles, Government of India, about 2.11 lakh handlooms are functioning in 1247 handloom weavers' co-operative societies and the remaining looms are outside the co-operative fold. The handloom weavers' cooperative societies mostly exist in Rural and Semi-Urban areas, where there is large concentration of handloom weavers.

\section{QUALITY OF FABRICS}

Generally, as any product in the market claims its quality, fabrics too have their own quality. The better the quality; on the customer perspective, the producer can expect more sales and on proprietor perspective, can fix a higher price. The manufacturer would always prefer to produce the highest quality goods within the shortest span of time. Till date, the process of identification, classification and correction of defects produced in a fabric; be it a handloom or machine weaved, is done manually. Humans are prone to errors; and more over the process involves a huge amount of caution during the process. A statistics proves that even the highest fabric inspector is capable of identifying only upto $70 \%$ of defects, whereas $30 \%$ remains unidentified, till it reaches the 
end-user. All these factors lead to a growing need for an automated fabric defect detection system which is the main objective of this paper.

\section{DEFECTS IN FABRICS}

In order to identify the most detrimental defects in textile fabrics, an industry survey was conducted to identify the most frequently occurring defects and the most costly defects as far as points were concerned. Data from leading fabric manufacturers was collected for their typical defects. Broken picks, harness drops, and start marks top the list of the most frequently occurring defects. Broken ends, broken picks, waste and coarse picks were the most costly defects. A wide variety of defects are represented; many defects are a direct cause of loom malfunction while others are from faulty yarns.

A broader classification is presented in this paper based on the survey to various industries located in and around Salem. The defects appearing in a silk fabric can be classified as

\section{Manufacturing or Weaving defects [2] and \\ 2. Handling Defects}

Both these are explained in detail with a special focus on silk fabrics.

\subsection{Manufacturing or Weaving defects}

\subsubsection{Missing Ends (Chira):}

A defect where one or more ends are missing in the fabric is called "Chira", which is shown in the figure 1 . It constitutes $10-50$ percent of total defects in loom static cloth

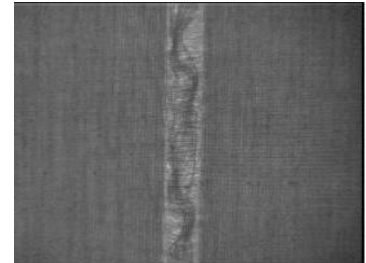

Fig 1: Missing Ends (Chira)

\subsubsection{Float (Jala) :}

Jala is formed when there is no proper interlacement of the warp and weft yarns over a certain area, as shown in the figure 2 .

Causes: Entanglement of adjoining ends in the regions between the heads and the fell of the cloth, knots with long tail ends, oozy and undersized warp, breakage of heald on running loom cause such entanglement

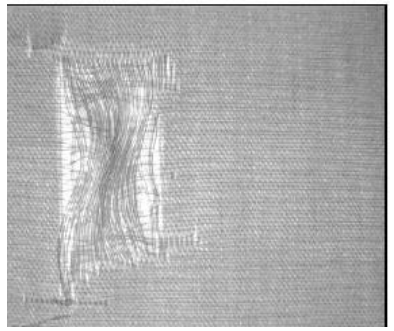

\subsubsection{Shuttle Smash}

Fig2: Float

The defect is caused when many ends break consequent to a shuttle trap.

Causes: Wrong timing of shedding, soft picking, insufficient checking of shuttle in the boxes, unbalanced shuttle, entanglement in the warp, damaged or broken picking.

\subsubsection{Temple Marks}

Yarns are distorted from their true paths and fine holes are caused near the sledge.

\subsubsection{Stains (Daghi)}

Caused by lubricants, due to lack of care on the part of operatives and the poor oiling and cleaning practices. A sample of a stained cloth is shown in the figure 3 .

\subsubsection{Hole in the cloth}

The cloth with any defect reformed, this portion becomes a hole and will be a waste.

Causes: Warp of weft float, shuttle smash, sharp edge on cloth roller front rest. Hard substance between layers of fabric in loom cloth roll. Coarse temples are used, Temple rolls not properly set. A simulated sample of a hole in a silk fabric is shown in the figure 4 .

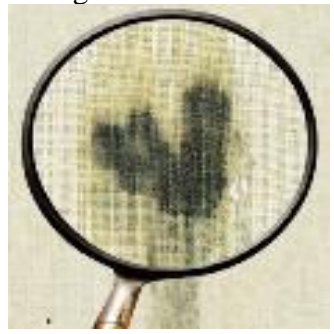

Fig 3: Stains in a Fabric

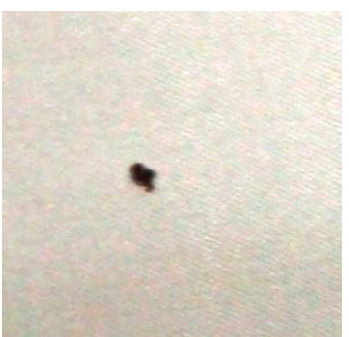

Fig 4: Hole in a dhothi

\subsubsection{Selvedge Defects}

i. Ends breaking at Selvedge

ii. Holes at selvedge (Temple Holes). Improper draw-in reed. Selvedges running slacker than body warp. Harsh picking. Big knots in selvedge yarn. Pins or rings or temple broken.

iii. Slack selvedge (loose selvedge). If count in selvedge yarn is too coarse.

iv. Corded Selvedge: Due to wrong drawing when the selvedge ends break.

v. Torn Selvedge: Left Hand temple in place of right hand temple. One side temple not rotating, shuttle tip burred or broken. Loose or cut emery sheet on the emery roll at the edge.

vi. Un-even selvedge

vii. Curling selvedge

viii. Loopy selvedge

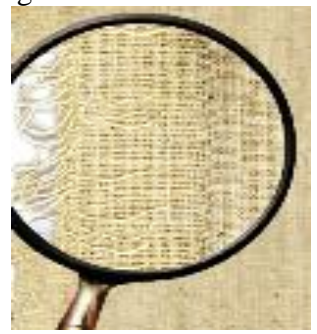

Fig 5: Selvedge Defects 


\subsection{Handling Defects}

These types of defects occur in a silk fabric mainly due to the poor handling of the silk cloths either by the customer or the sales person (see figure 6). Most of such defects are not rectifiable.

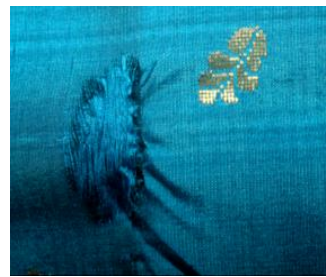

Fig 6: Handling Defect

\section{Defect Detection System}

Defect inspection in fabrics is of great importance to improve the quality [26]. Digital image processing methods have been developed for defect detection for past few years, and an elaborate discussion on the various techniques are available in $[3,4,5,6 \& 7]$.

\section{1 Texture Analysis}

Texture analysis is used in many applications field like textile industrial, agricultural, remote sensing and biomedical [8] surface inspection. For example, identification of defects in textile fabrics, disease identification in human organs, classification and segmentation of satellite imagery, segmentation of textured regions in document analysis, and many more. The major issues in the real world textures are not uniform due to changes in orientation, size or other visual appearance and also the measurement of texture features are very high computational complexity.

Texture is the repetition of image patterns, which may be perceived as being directional or non-directional, smooth or rough, coarse or fine, regular or irregular, etc.

\section{2 Fabric Texture Analysis}

The fabric texture usually is made of the repetition arrangement of warp and weft and so image processing techniques can be used for defect detection [14]. Textile Fabric materials are used to prepare different categories and types of fabric products in the textile industry. Natural fabric and synthetic fabric are the two different classification of textile fabric. Synthetic fabrics are fairly new and have evolved with the continuous growth in textile industry.

Some sensor and microcontroller based designs have been carried out in [20, $21 \& 23]$, which limits its defect detection to only a few, when compared to that of the various defects as listed out in [2]. Basic Fourier transformation can be used for modeling the woven fabrics as in $[9,19 \& 22]$. In some cases, a motif based defect detection in a patterned fabric [10]. Optimal Gabor Filters can be used for detecting the defects in the fabrics [11], along with textural models [24] and structural approaches [25].

With the advancements in the Wavelet Transforms, some researchers have experimented in using them for fault segmentation [12, 13]. This paper deals with the texture features in an image which can be further extended and used for the classification methodology as discussed in [14]. An experiment was also made by Paramasivam and et.al in $[16$ \& 17] to implement the DWT modeled for fabric defect detection in a NIOS II Processor.

\subsubsection{Feature Extraction MRCSF:}

MRCSF [18 \& 27] refers to Multi Resolution Combined Statistical and Spatial Frequency Method. It is a combination of first order statistical features (like energy, mean, standard deviation and variance) second order statistical features (MRFM, GLCM) [28 \& 30] and Spatial Frequency for Multi Resolution Analysis

\subsubsection{Markov Random Field Matrix (MRFM)}

Markov Random Field theory is a branch of probability theory for analyzing the spatial or contextual dependencies of physical phenomena. $\mathrm{F}$ is said to be a Markov random field with respect to a neighborhood system $\mathrm{N}$ if and only if the following conditions are satisfied [29]

1. Positivity : $\mathrm{P}(\mathrm{F})>0$ for all $\mathrm{F}$.

2. Markovianity : $\mathrm{P}(\mathrm{F})$ all points in the lattice except $\mathrm{P}(\mathrm{F}(\mathrm{i}))$ neighbors of (i).

3. Homogeneity : $\mathrm{P}((\mathrm{F}(\mathrm{i}))$ neighbors of (i) depends only on the configuration of neighbors and its translation invariant.

This is a parametric approach where the texture is modeled as a Markov Random Field. First the type of neighborhood is chosen and then, the parameters on which functions depend characteristic the texture. These are called Markov Parameters. MRF Matrix is constructed from the 9 MRF parameters $(\beta 1, \beta 2, \beta 3, \beta 4, \gamma 1, \gamma 2, \gamma 3, \gamma 4$ and $\xi)$.

MRF parameters are extracted in a $3 \times 3$ size matrix from an image at gray-level. The procedure consists of the following steps:

(1) Find the relationship between the center pixel and its nearest neighbors in the $3 \times 3$ matrix by making use of the equations from (1) to (34).

(2) Obtain 9 different MRF parameters from the 8 neighborhood system.

(3) The parameter of $\beta$ depends on two pixel relationships, $\gamma$ depends on three pixel relationship and $\xi$ depends on four pixel relationship.

(4) MRF parameter matrix [M] output contains 9 parameters so the size is $1 \times 9$. Obtain the transpose of $\mathrm{M}$ matrix $\left[\mathrm{M}^{\mathrm{T}}\right]$ and multiply it with $M$ matrix. It provides $9 \times 9$ size MRF matrix.

(5) Obtain MRF features from the MRF matrix.

\begin{tabular}{|c|c|c|}
\hline $\boldsymbol{i}_{1}$ & $\boldsymbol{i}_{2}$ & $\boldsymbol{i}_{3}$ \\
\hline $\boldsymbol{i}_{8}$ & $\boldsymbol{i}$ & $\boldsymbol{i}_{4}$ \\
\hline $\boldsymbol{i}_{7}$ & $\boldsymbol{i}_{6}$ & $\boldsymbol{i}_{5}$ \\
\hline
\end{tabular}

Fig 7: Pixel i and its eight neighbors in the second order neighborhood system

Nine different MRF parameters are extracted from the eight neighborhood system shown in Figure 7.

$$
M=\left[\beta_{1}, \beta_{2}, \beta_{3}, \beta_{4}, \gamma_{1}, \gamma_{2}, \gamma_{3}, \gamma_{4}, \xi\right]
$$

The procedure for determining the values for $\beta_{1}, \beta_{2}$, $\beta_{3}, \beta_{4}, \gamma_{1}, \gamma_{2}, \gamma_{3}, \gamma_{4}$ and $\xi$ is given below.

$$
\beta_{1}=\beta_{11}+\beta_{12}
$$

where 
$\beta_{11}= \begin{cases}1 & \text { if } f_{i}=f_{8} \\ 0 & \text { otherwise }\end{cases}$

$\beta_{12}= \begin{cases}1 & \text { if } f_{i}=f_{4} \\ 0 & \text { otherwise }\end{cases}$

$\beta_{2}=\beta_{21}+\beta_{22}$

where

$\beta_{21}= \begin{cases}1 & \text { if } f_{i}=f_{2} \\ 0 & \text { otherwise }\end{cases}$

$\beta_{22}= \begin{cases}1 & \text { if } f_{i}=f_{6} \\ 0 & \text { otherwise }\end{cases}$

$\beta_{3}=\beta_{31}+\beta_{32}$

where

$\beta_{31}= \begin{cases}1 & \text { if } f_{i}=f_{1} \\ 0 & \text { otherwise }\end{cases}$

$\beta_{32}= \begin{cases}1 & \text { if } f_{i}=f_{5} \\ 0 & \text { otherwise }\end{cases}$

$\beta_{4}=\beta_{41}+\beta_{42}$

where

$\beta_{41}= \begin{cases}1 & \text { if } f_{i}=f_{3} \\ 0 & \text { otherwise }\end{cases}$

$\beta_{42}= \begin{cases}1 & \text { if } f_{i}=f_{7} \\ 0 & \text { otherwise }\end{cases}$

$\gamma_{1}=\gamma_{11}+\gamma_{12}+\gamma_{13}$

where

$\gamma_{11}= \begin{cases}1 & \text { if } f_{i}=f_{1}=f_{8} \\ 0 & \text { otherwise }\end{cases}$

$\gamma_{12}= \begin{cases}1 & \text { if } f_{i}=f_{2}=f_{4} \\ 0 & \text { otherwise }\end{cases}$

$\gamma_{13}= \begin{cases}1 & \text { if } f_{i}=f_{5}=f_{6} \\ 0 & \text { otherwise }\end{cases}$

$\gamma_{2}=\gamma_{21}+\gamma_{22}+\gamma_{23}$ where

$\gamma_{21}= \begin{cases}1 & \text { if } f_{i}=f_{1}=f_{2} \\ 0 & \text { otherwise }\end{cases}$

$\gamma_{22}= \begin{cases}1 & \text { if } f_{i}=f_{6}=f_{8} \\ 0 & \text { otherwise }\end{cases}$

$\gamma_{23}= \begin{cases}1 & \text { if } f_{i}=f_{4}=f_{5} \\ 0 & \text { otherwise }\end{cases}$

$\gamma_{3}=\gamma_{31}+\gamma_{32}+\gamma_{33}$

where

$\gamma_{31}= \begin{cases}1 & \text { if } f_{i}=f_{2}=f_{8} \\ 0 & \text { otherwise }\end{cases}$

$\gamma_{32}= \begin{cases}1 & \text { if } f_{i}=f_{3}=f_{4} \\ 0 & \text { otherwise }\end{cases}$

$\gamma_{33}= \begin{cases}1 & \text { if } f_{i}=f_{6}=f_{7} \\ 0 & \text { otherwise }\end{cases}$

$\gamma_{4}=\gamma_{41}+\gamma_{42}+\gamma_{43}$

where

$\gamma_{41}= \begin{cases}1 & \text { if } f_{i}=f_{2}=f_{8} \\ 0 & \text { otherwise }\end{cases}$

$\gamma_{42}= \begin{cases}1 & \text { if } f_{i}=f_{3}=f_{4} \\ 0 & \text { otherwise }\end{cases}$

$\gamma_{43}= \begin{cases}1 & \text { if } f_{i}=f_{6}=f_{7} \\ 0 & \text { otherwise }\end{cases}$

$\xi=\xi_{11}+\xi_{12}+\xi_{13}+\xi_{14}$

where

$\xi_{11}= \begin{cases}1 & \text { if } f_{i}=f_{1}=f_{2}=f_{8} \\ 0 & \text { otherwise }\end{cases}$

$\xi_{12}= \begin{cases}1 & \text { if } f_{i}=f_{2}=f_{3}=f_{4} \\ 0 & \text { otherwise }\end{cases}$

$\xi_{13}= \begin{cases}1 & \text { if } f_{i}=f_{6}=f_{7}=f_{8} \\ 0 & \text { otherwise }\end{cases}$ 
$\xi_{14}= \begin{cases}1 & \text { if } f_{i}=f_{4}=f_{5}=f_{6} \\ 0 & \text { otherwise }\end{cases}$

and $\mathrm{M}\left(f_{\mathrm{i}}, f N_{\mathrm{i}}\right)=\left[{ }^{x}\left(f_{\mathrm{i}}, f_{\mathrm{i} 4}\right)+x_{\left(f_{\mathrm{i}}, f_{\mathrm{i} 8}\right),} x_{\left(f_{\mathrm{i}}, f_{\mathrm{i} 2}\right)+}\right.$

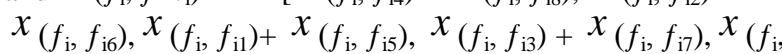

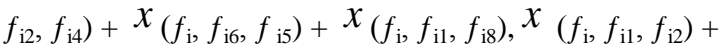

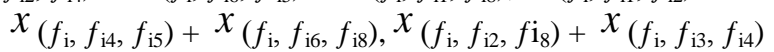

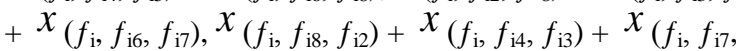

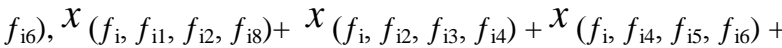

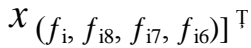

$$
M R F M=M^{T} M\left(f_{i}, f_{N i}\right)
$$

The RHS of the first row in the equation (35) corresponds to the potential weighted by $\beta_{1}$ and $\beta_{2}$, the second row by $\beta_{3}$ and $\beta_{4}$, the third to sixth by $\gamma_{1 \ldots \ldots . .} \gamma_{4}$, and the last two rows by $\xi_{1}$.

The parameters to be found in extracting MRFM features are $\beta 1, \beta 2, \beta 3, \beta 4, \gamma 1, \gamma 2, \gamma 3, \gamma 4$ and $\xi$. The way of MRFM parameters estimation using second order system is shown in Figure 3.9
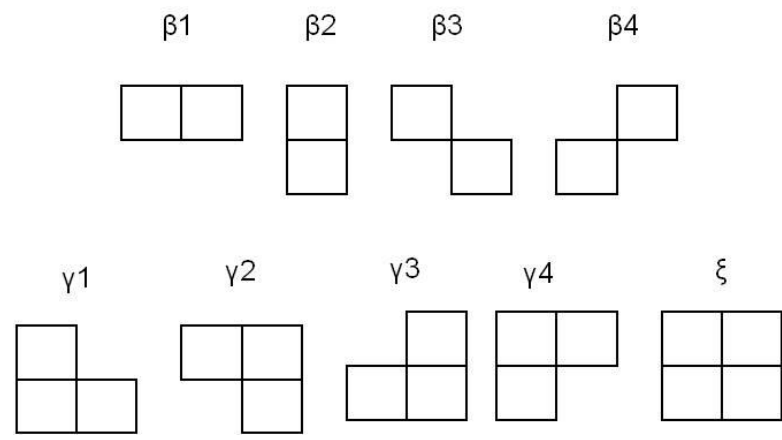

Fig 7 MRFM parameter estimation using second order system

\subsubsection{Gray Level Co-occurrence Matrix (GLCM)}

The Gray Level Co-occurrence Matrix [15] C[i, j] is defined by the first specifying a displacement vector $\mathrm{d}=(\mathrm{dx}, \mathrm{dy})$ and counting all pairs of pixels separated by $d$ having gray levels $i$ and $\mathrm{j}$. Count all pairs of pixels in which the first pixel has the value of $i$ and its matching pair displaced from the first pixel by $d$ has a value of $j$, and also enter this count in the $i^{\text {th }}$ row and $j^{\text {th }}$ column of the matrix

$$
\begin{aligned}
& \text { 3.2.3 1. Energy / Uniformity } \\
& \qquad f_{1}=\sum_{i, j}^{N} C_{i, j}^{2}
\end{aligned}
$$

Where $\mathrm{f} 1$ is the energy feature and $\mathrm{C}_{\mathrm{i}, \mathrm{j}}$ is the co-occurrence matrix.

\subsubsection{Maximum Probability}

This property gives an indication of the strongest response to the texture pattern.

$$
\sum_{i=0}^{N-1} \sum_{j=0}^{M-1} \operatorname{Max} C_{i, j}
$$

\subsubsection{Element difference moment of order $k$}

This descriptor has a relatively low value when high values of co-occurrence matrix are near the main diagonal because the difference is very small.

$$
\sum_{i=0}^{N-1} \sum_{j=0}^{M-1} C_{i, j}(i-j)^{k}
$$

3.2.3.4. Inverse element difference moment of order $k$

$$
\sum_{i=0}^{N-1} \sum_{j=0}^{M-1} \frac{c_{i, j}}{(i-j)^{k}}
$$

This has an opposite effect of previous defined one.

\section{Entropy}

$$
\sum_{i=0}^{N-1} \sum_{j=0}^{M-1} C_{i, j} \log \left(c_{i, j}\right)
$$

Entropy is a measure of randomness, achieving its highest value when all elements of co-occurrence matrix are equal.

\subsubsection{Spatial Frequency}

The spatial frequency [18] is used to measure the overall information level in the regions. This is computationally simple and efficient and also can be used in real time applications. The spatial frequency for an $\mathrm{M} \times \mathrm{N}$ block of an image is calculated as follows

$$
\begin{aligned}
& S F=\sqrt{(R F)^{2}+(C F)^{2}} \\
& R F=\sqrt{\frac{1}{N^{2}} \sum_{m=1}^{N} \sum_{n=2}^{N}[F(m, n)-F(m, n-1)]^{2}} \\
& C F=\sqrt{\frac{1}{N^{2}} \sum_{m=1}^{N} \sum_{n=2}^{N}[F(m, n)-F(m-1, n)]^{2}}
\end{aligned}
$$

Where RF and $\mathrm{CF}$ are the row frequency and column frequency respectively. When the images get more blurred, the spatial frequency also gets reduced accordingly. Higher the value of spatial frequency, higher will be the contrast and quality of the image. In each sub band, individual pixels or group of pixels of the wavelet transform of the images are compared using spatial frequency (SF) that serves as a measure of activity at that particular scale and space. Other examples of such measures are absolute values of the pixel gray values, maximum absolute gray value of the group of pixels and the variance.

\subsubsection{GLWM}

In this method, the local texture information for a given pixel and its neighborhood is characterized by the corresponding texture unit and the global textural aspect of an image is revealed by its texture spectrum. This method extracts the textural information of an image with a more complete respect 
of texture characteristics. In GLWM [30] method instead of thres-holding the image transforming is made with neighborhood to a texture unit with the texture unit number under the ordering way as shown in figure 2 .

A brief overview of the process of defect detection for the proposed method is shown in figure 8 .

1. Feature Extraction of original image: This is the initial task in which the original non-defective reference samples are collected and their features are extracted using appropriate algorithm and stored in a database. Before feature extraction the sample images are wavelet transformed so that the samples are localized in both time and frequency. MRCSF Features like mean, standard deviation, energy, entropy, spatial frequency, Multi Resolution Markov Random Field Matrix and Gray Level Co occurrence Matrix (GLCM) for both the reference fabric and the fabric to be tested were extracted using MATLAB 7.5 and hence compared for classification. All the above mentioned steps are done using MATLAB Image Processing toolbox and Database Toolbox.

2. Capturing and Feature extraction of test sample: This part comes under the classification stage where the test samples are captured using a digital camera which is attached to a shaft which moves over the entire sample. The movement of the shaft is controlled by embedded system which employs a microcontroller. After capturing the sample images the feature are extracted in the same way as in the case of original image and stored in the library.

3. Comparison with Library: In this stage the stored features of the original image and the test sample are compared using the nearest neighborhood algorithm. The test samples are classified as defective or non-defective based on the comparison results.

4. Indication of the Defects: The obtained defect is analyzed for its type using the available database of defects and hence the defect type is displayed on the screen. The location of the defect is also displayed on the screen for the ease of the user.

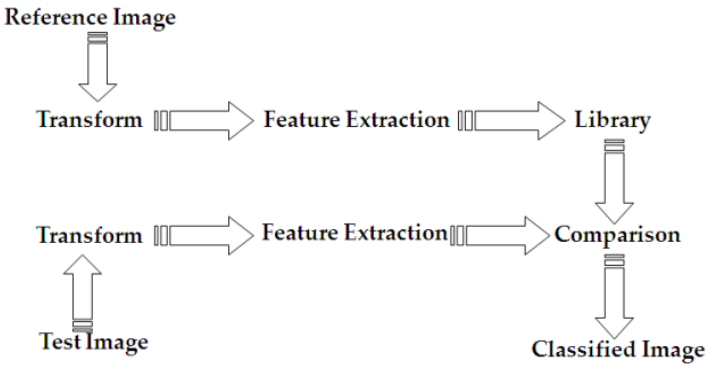

Fig 8: Defect detection system

\section{RESULTS}

Due to the absence of standard databases for the silk fabrics, a database was created with 25 reference images. These reference images were used for training the GUI designed with the algorithm. Each of these images are of the $512 \times 512$ size.

Various images of silk fabrics with defects as discussed in section 3.1 and 3.2 were given to the system to check if the system is capable of finding the defects present in the image.

Some of the snapshots of the same are shown in figure 9.
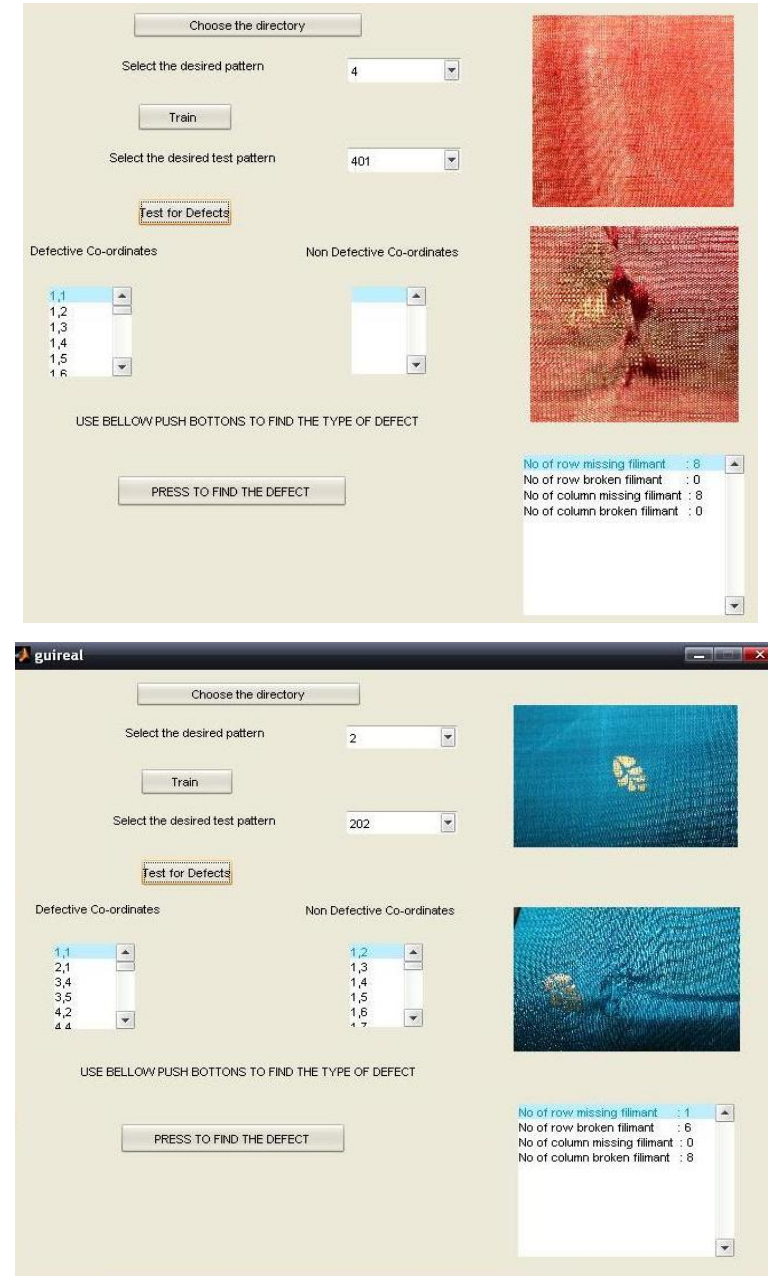

Fig 9 Snapshots of the GUI used for defect detection in silk sari

\section{CONCLUSION}

The proposed work succeeded in classifying the fabrics with repeated patterns as defective or non defective based on the MRCSF that was performed. MRCSF is a combination of first order statistical properties like mean, energy, variance and entropy, second order statistical properties like Markov Random Field Matrix, Gray level co-occurrence matrix combined with spatial frequency of Multi resolution analysis. Location and type of the defects are also identified. GLCM approach is a combination of wavelet and co-occurrence matrix features. It provides a good success rate of classification for fabrics. MRMRFM based approach is a combination of wavelet and MRFM features. In Real time Fabric the size of the sub window reduces the defect identification rate also reduces. The Classification rate of real time fabric achieved $96.6 \%$ for 25 samples.

Hardware implementation of the testing process can also be carried out keeping the cost constraint in mind. As a part of future work the same algorithm can be implemented in handloom cottage silk industries using DSP processor whenever necessary and hence forth calculate the computation time of the proposed method.

\section{ACKNOWLEDGEMENT}

The authors would like to thank the All India Council of Technical Education (AICTE), New Delhi for the financial support and the Sona Trust for the providing the financial and 
moral support towards the research work. They also wish to thank their colleagues of the SONA SIPRO Research team of Sona College of Technology, Salem and their family members for many fruitful discussions. The authors wish to place their special thanks to Dr.S.Jayaraman, Professor, Dean and Head of the Department of Electronics and Communication Engineering, Sona College of Technology, Salem, Tamil Nadu, India and Dr.V.Palanisamy, Principal, Info Institute of Engineering, Coimbatore, Tamil Nadu, India. The Authors also wish to place their special thanks to the authorities of Indian Institute of Handloom Technology, Salem, Tamil Nadu, India; Co-optex, Salem, Tamil Nadu, India and other handloom cottage silk industries in and around Salem, Tamil Nadu, India for extending their co-operation capturing nondefective and defective images.

\section{REFERENCES}

[1] Diagnostic Study of Trichy Handloom Cluster under the MSME Clusters

[2] R.C.M.Reddy, I.A.S, Member Secretary, Textiles Committee, Ministry of Textiles, "A catalogue on woven fabric defects and visual inspection", Quality Appraisal and Export Promotion, \& Market Research Wings, Textiles Committee, MUMBAI - 400018.

[3] Kumar, A , "Computer-Vision-Based Fabric Defect Detection: A Survey", IEEE Transactions on Industrial Electronics, vol. 55 , no. 1, Page(s): 348 - 363, 2008, Digital Object Identifier: 10.1109/TIE.1930.896476

[4] R. T. Chin and C. A. Harlow, "Automated visual inspection: A survey," IEEE Transactions in Pattern Analysis Mach. Intelligence, vol. PAMI-4, no. 6, pp.557-573, Jun. 1982.

[5] R. T. Chin, "Automated visual inspection: 1981 to 1987," Computer Vision, Graphics, and Image Processing, vol. 41, no. 3, pp. 346-381, Mar. 1988.

[6] T. S. Newman and A. K. Jain, "A survey of automated visual inspection," Computer Vision Image Understanding, vol. 61, no. 2, pp. 231-262, Mar. 1995.

[7] A. D. H. Thomas, M. G. Rodd, J. D. Holt, and C. J. Neill, "Real-time industrial inspection: A review," Real- Time Imaging, vol. 1, no. 2, pp. 139-158, Jun. 1995

[8] Sabeenian R.S. and Palanisamy V., 'Texture Based Medical Image Classification of Computed Tomography images using MRCSF', Published in the International Journal of Medical Engineering and Informatics (IJMEI, Inderscience), Vol. 1, No. 4 pp 459-472

[9] Escofet J., Milan M.S. and Rallo M. (2001), 'Modelling of Woven Fabric structures based on Fourier Image analysis', Appl. Opt., Vol. 40, pp. 6170-6176.

[10] Henry Y.T.N., Grantham K.H.P., Nelson H.C.Y. (2008), 'Motif-Based defect detection for patterned fabric', Pattern Recognition Letters, Vol. 41, No. 6, pp. 18781894.

[11] Mak K.L. and Peng P. (2006), 'Detecting Defects in Textile Fabrics with Optimal Gabor Filters', Proceedings of World Academy of Science, Engineering and Technology, Vol. 13, pp. 75-80.

[12] Arivazhagan S. and Ganesan L. (2003), 'Texture Classification using Wavelet Transform', Pattern Recognition Letters, Vol. 24, Nos. 9-10, pp. 1513-1521.
[13] Arivazhagan S., Ganesan L. and Bama S. (2006), 'Fault segmentation in fabric images using Gabor wavelet transform', International Journal of Machine Vision and Applications, Vol. 16, No. 6, pp. 356-363.

[14] Haralick R.M., Shangmugam K. and Dinstein L. (1973), 'Textural Features for Image Classification', IEEE Trans. Systems, Man, and Cybernetics, Vol. 3, pp. 610-621.

[15] Sabeenian R.S. and Palanisamy V., 'Comparison of Efficiency for Texture Image Classification Using MRMRF and GLCM Techniques', Published in International Journal of Computers Information Technology and Engineering (IJCITAE), Vol. 2, No. 2, December 2008, pp. 87-93.

[16] Sabeenian R.S and M.E.Paramasivam, 'Handloom Silk Fabric Defect Detection using First order Statistical Features on a NIOS II Processor' Published in the Springer International Conference on Advances in Information and Communication Technologies ICT 2010 held on September 2010 at Cochin, India, 2010, Volume 101, Part 3, pp 475-477.

[17] M.E.Paramasivam and R.S.Sabeenian, "A Soft Core Processor based Implementation of DWT for Identifying Defects in Fabric", in the proceedings of 2nd National Conference on Signal Processing Communications and VLSI Design (NCSCV '10), 7th and 8th May 2010, held by Department of Electronics and Communication Engineering Anna University Coimbatore.

[18] Sabeenian R.S. and Palanisamy V., 'Texture Based Weed Detection Using Multi Resolution Combined Statistical and spatial Frequency', Published in the International Journal of World Academy of Science, Engineering and Technology, WASET Vol. 40, April 2009, pp 87-93.

[19] Wood E.I. (1990), 'Applying Fourier and associated transform to pattern characterization in textiles', Textile Research Journal Vol. 60, pp. 212-220

[20] Tamnun E.M., Fajrana Zebin Eishita, Ahmed Ridwanul Islam and Md Jahangi Alam (2008), 'Real Time Automated Fabric Defect Detection System using Microcontroller', Journal of Theoretical and Applied Information Technology, Vol. 3, No. 1, pp. 42-50.

[21] X. F. Zhang and R. R. Bresee, "Fabric defect detection and classification using image analysis," Textile Research Journal, vol. 65, no. 1, pp. 1-9, 1995.

[22] E. J. Wood, "Applying fourier and associated transforms to pattern characterization in textiles," Textile Research Journal, vol. 60, no. 4, pp. 212-220, 1990.

[23] M. Unser and F. Ade, "Feature extraction and decision procedure for automated inspection of textured materials," Pattern Recognition Letters, vol. 2, no. 2, pp. 181-191, 1984.

[24] F. S. Cohen, Z. Fan, and S. Attali, "Automated inspection of textile fabrics using textural models," IEEE Transactions on Pattern Analysis and Machine Intelligence, vol. 13, no. 8, pp. 803-808, 1991.

[25] J. Chen and A. K. Jain, "A structural approach to identify defects in textured images," in Proceedings of IEEE International Conference on Systems, Man, and Cybernetics (SMC '88), vol. 1,pp. 29-32, Beijing, China, August 1988. 
[26] A. Atalay, "Automated defect inspection of textile fabrics using machine vision techniques," M.S. thesis, Bogazici University, Istanbul, Turkey, 1995.

[27] R.S.Sabeenian and M.E.Paramasivam, "Defect detection and Identification in Textile Fabrics using Multi Resolution Combined Statistical and spatial Frequency Method", in the proceedings of 2010 IEEE $2^{\text {nd }}$ International Advance Computing Conference (IACC),vol., no., pp.162-166, 19-20 Feb. 2010 doi: 10.1109/IADCC.2010.5423017,

[28] R.S. Sabeenian and P.M. Dinesh, "Texture Image Classification Using Gray Level Weight Matrix (GLWM)" in the Proceedings of AIM 2011, CCIS 147, pp. 263-266, Springer-Verlag Berlin Heidelberg 2011

[29] R.S.Sabeenian, M.E.Paramasivam and P.M.Dinesh, "Detection and Location of Defects in Handloom Cottage Silk Fabrics using MRMRFM \& MRCSF", International Journal of Technology And Engineering System (IJTES): Jan -March 2011- Vol.2.No.2. pp 172 176.

[30] R.S.Sabeenian, M.E.Paramasivam and P.M.Dinesh, "Handloom Silk Defect Recognition and Categorization using Gray Level Weight Matrix \& Multi Resolution Combined Statistical and Spatial Frequency Method" published in the Proceedings of ICCAC 2011, held at Cape Institute of Technology, Kanyakumari

\section{AUTHORS PROFILE}

Dr.R.S.Sabeenian is currently working as an Professor in ECE Department in Sona College of Technology, Salem, Tamil Nadu, India. He received his Bachelors in Engineering from Madras University and his Masters in Engineering in Communication Systems from Madurai Kamaraj University. He received his Ph.D Degree from Anna University, Chennai in the area of image processing. He is currently heading the research group named Sona SIPRO (SONA Signal and Image PROcessing) centre located at the Advanced Research Centre in Sona College of Technology, Salem. His areas of interest include texture analysis, texture classification and pattern recognition. He has published around 33 papers in various international, national journals and conferences. He has also published around seven books. He is a reviewer for the journals of IET, UK and ACTA Press Singapore. He received the - Best Faculty Award among Tamil Nadu, Karnataka and Kerala states for the year 2009 given by the Nehru Group of Institutions, Coimbatore, "ISTE Rajarambapu Patil National
Award" for promising Engineering Teacher for Creative work done in Technical Education (Colleges) for the year 2010 and "IETE Biman Behari Sen Memorial Award" for outstanding contributions in the emerging areas of Electronics and Telecommunication with emphasis on R\&D for the year 2011. $\mathrm{He}$ has also received a Project grant from the All India Council for Technical Education and from the Tamil Nadu State Council for Science and Technology for his research.

M.E.Paramasivam, is currently working as the Research Staff in Sona Signal and Image PROcessing Research Centre located at the Advanced Research Centre, Sona College of Technology, Salem. He is also the Assistant Professor in the ECE department of Sona College of Technology, Salem.

$\mathrm{He}$ completed his Bachelors in Electronics and Communication Engineering in the year 2005. Later he was selected on an all India basis for pursing the Advanced Post Graduate Diploma in VLSI Design, from VEDANT Center, of Semiconductor Complex Ltd., A Govt. of India Enterprise located at Chandigarh in the year 2006. He also holds a Diploma in Management, Post Graduate Diploma in Management, Post Graduate Diploma in Human Resource Management and Masters in Management. He is a certified Altera Tools trainer, certified by Altera Semiconductors. USA. He also holds a Masters Degree in Engineering with specialization in VLSI Design in the year 2011. He is currently pursuing his Doctoral Research in the area of Digital Image Processing. $\mathrm{He}$ has published and presented 15 research papers, both in technical and non-technical areas, at various conferences conducted around the nation. To his credit he has also published two papers in International Journal. He has written a book on "Electric Circuits and Electron Devices" in 2009. . He has also received a Project grant from the All India Council for Technical Education under the Research Promotion Scheme (RPS).

P.M.Dinesh is currently working as Assistant Professor of ECE Department, at Sona College of Technology, Salem. He is also a Team member of Sona SIPRO, Sona Signal and Image PROcessing Research Centre. He completed his Bachelors in Engineering in Electronics and Communication Engineering in the year 2005 and Masters in Engineering in VLSI Design in the year 2011. He is currently pursuing his Doctoral Research in the area of Image Processing. His areas of interest include pattern recognition and texture analysis using MATLAB. He has published ten papers in leading conferences and journals in the country. 